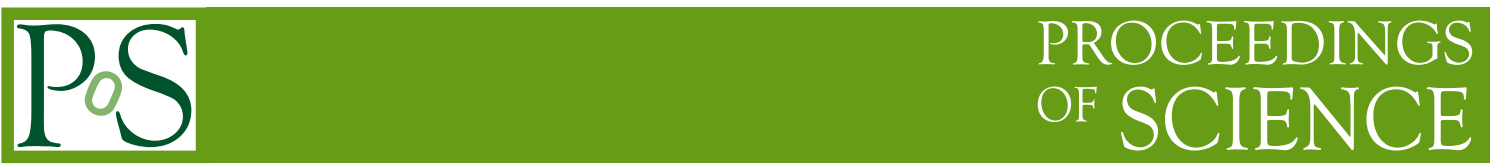

\title{
Highlights of EW and QCD results at the LHC
}

\author{
Javier Jiménez Peña* on behalf of the ATLAS and CMS collaborations \\ Max-Planck-Institut für Physik (Werner-Heisenberg-Institut), \\ Föhringer Ring 6, D-80805 München, Germany. \\ E-mail: javier.jimenez.pena@cern.ch
}

Using collision data from the LHC, the ATLAS and CMS collaborations have extensively tested the Standard Model of particle physics with a wide programme of physics analyses. This document highlights a few recent analyses targeting electroweak and quantum chromodynamics results to give the reader an insight into the different approaches and techniques currently exploited. The highlighted analyses can roughly be classified into four different categories: the measurement of differential cross-sections, the search for rare processes, the measurement of particle properties and the search for new physics interpreted within an effective field theory extension of the Standard Model.

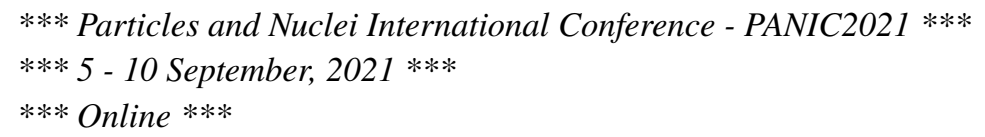

${ }^{*}$ Speaker 


\section{Introduction}

The Standard Model (SM) of particle physics is the most successful theory describing our current understanding of subatomic particles and their interactions to date. Two of the main ingredients of the SM are the quantum chromodynamics (QCD) and the electroweak (EW) theories that describe the strong and the electromagnetic and weak interactions, respectively. Using protonproton collision data from the LHC [1], the ATLAS [2] and CMS [3] collaborations have extensively tested the SM predictions in a wide range of processes, covering more than nine orders of magnitude in cross-sections, from frequent processes such as jet production to rare ones such as photon-induced $\mathrm{W}$ boson pair production. Precise experimental results allow to test higher-order calculations in QCD and EW theories. This is of vital importance since the new physics reachable by the LHC will likely appear as small deviations from the SM predictions. In what follows, a selection of recent QCD and EW results from the ATLAS and CMS collaborations is presented. These were obtained using proton-proton collision data from the Run-2 of the LHC, with a centre-of-mass energy of $13 \mathrm{TeV}$. The selected analyses can be classified into the four categories mentioned above. A record of the full talk, where a more extensive summary was done, is available [4].

\section{Differential cross-section measurements}

Differential cross-section measurements provide a powerful tool to test the SM, allowing the comparison of data and theoretical predictions for numerous observables like the transverse momentum of a particle, providing the needed information to improve the theoretical predictions. The ATLAS and CMS collaborations perform differential measurements in a wide range of processes, covering both frequent and rare processes such as jet or $t Z q$ production, respectively.

Using a partial $13 \mathrm{TeV}$ dataset, the CMS collaboration has measured the double-differential inclusive jet cross-section as a function of the jet transverse momentum and the absolute jet rapidity [5]. The measured distributions, shown in Figure 1, were unfolded to particle level, allowing a comparison with perturbative QCD predictions at next-to-leading order (NLO), next-toleading order with next-to-leading logarithmic corrections (NLO+NLL) and next-to-next to leading order (NNLO). Among other things, these data were used together with a previous multi-differential $t \bar{t}$ cross-section measurement and HERA data to improve the predicted parton distribution functions (PDF) of quarks and gluons inside the proton. Figure 1 shows the PDF for the valence u-quarks and the gluons as functions of the proton momentum fraction $x$. The new PDF predictions have reduced uncertainties compared to the ones based on HERA data only.

The ATLAS collaboration has performed a measurement of prompt photon-pair production in proton-proton collisions using the full Run-2 dataset [6]. Prompt photon-pairs are usually the main background to analyses searching for, or studying the properties of particles decaying into a pair of photons, such as the Higgs boson. Therefore, understanding the photon-pair production is of vital importance experimentally. Additionally, photon-pair production in hadron colliders involves intricate, strong-interaction dynamics, what turns this measurement into an important probe of the strong force. One of the main challenges of the measurement is a precise determination of nonprompt photon-pairs from hadron decays. A data-driven technique is used for that purpose, where a total of 15 control regions (CR) are defined by inverting the photons isolation and identification 

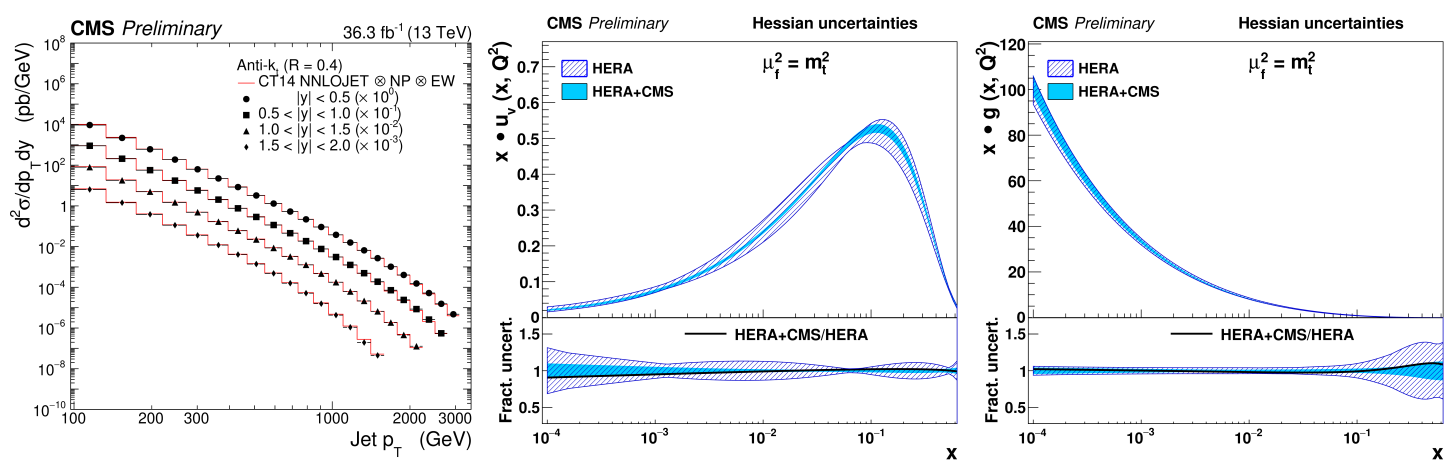

Figure 1: CMS: The measured cross-section of jets as a function of the jet $p_{\mathrm{T}}$ for various $|y|$ ranges (left) and the newly obtained parton distribution functions for $u$-quarks (central) and gluons (right) [5].

requirements of the signal region (SR) as shown in Figure 2. The background subtracted data is unfolded to particle level in a fiducial phase space similar to the experimental acceptance and is compared to theoretical predictions from various MC simulations and fixed-order calculations. The importance of higher-order QCD corrections is visible in Figure 2, where both the integrated and the differential (as a function of the photon pair transverse momenta) fiducial cross-sections are shown. Only the NNLO QCD and SHERPA predictions are able to describe the data. Of particular interest is the differential transverse momenta of the photon-pair, which vanishes at $\mathrm{LO}$ due to momentum conservation. Only when additional particles are radiated (described by higher-order calculations) this quantity is non-zero.
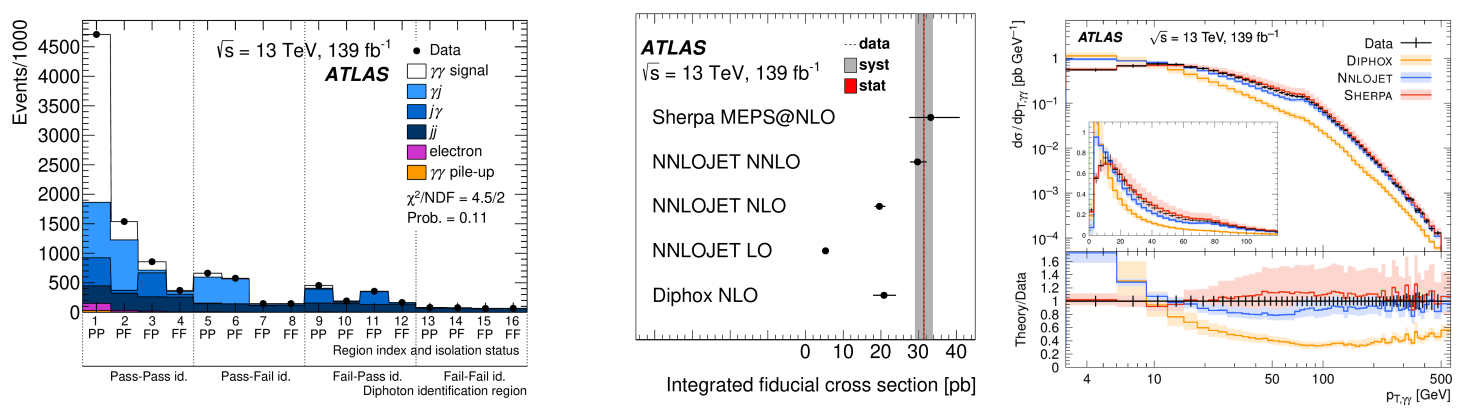

Figure 2: ATLAS: Event yields in the signal and control regions (left). The measured inclusive cross-section of diphoton production (central) and the differential diphoton cross-section as a function of $p_{\mathrm{T}}$ (right), both compared to various theoretical predictions [6].

The last example is the differential cross-section of $t Z q$ production, which was measured for the first time by the CMS collaboration using the full $13 \mathrm{TeV}$ dataset [7]. This process is of great interest as it involves $t Z, t W b$ and $W W Z$ couplings, and at the same time has a low QCD background, making it an important probe of the EW theory. This measurement makes extensive use of machine learning algorithms to make best use of the small sample size. In a first step, a boosted decision tree (BDT) discriminates signal from background in three independent signal regions according to the number of jets and b-jets (Figure 3). Afterwards, a multiclass neural network (NN) splits selected events in five output nodes to distinguish $t Z q$ events from $t \bar{t} Z$ and various background processes. This provides an improved isolation of $t Z q$ events compared to an analysis using a 
binary classifier. For the differential measurements, the $t Z q$ output node of the $\mathrm{NN}$ is split into three or four sub-regions depending on the measured observable. As an example, Figure 3 shows the differential cross-section as a function of the top-quark polarisation $\operatorname{angle}, \cos \left(\theta_{\text {pol }}^{*}\right)$, which is used in the analysis to determine the top-quark spin asymmetry.
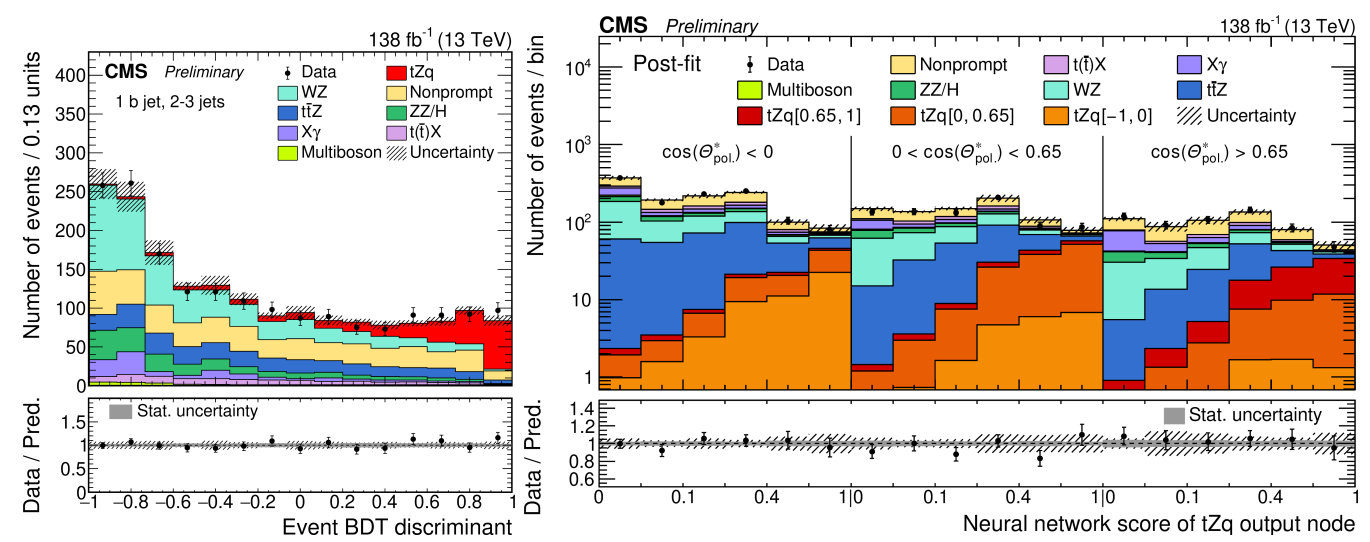

Figure 3: CMS: The output of the discriminating boosted decision tree for the one $b$-jet signal region (left) and the output of the multiclass neuronal network for the signal node for various ranges of $\cos \left(\theta_{\mathrm{pol}}^{*}\right)$ (right) [7].

\section{Rare processes}

Although they have small cross-sections that make them harder to detect, the measurement of rare processes constitutes an important test of the SM. The production of these kind of processes is in many cases proportional to less-tested couplings, as the self interaction of gauge-bosons through triple- or quartic-gauge couplings. Deviations in the predicted cross-sections of rare events could indicate new physics.

For the first time, the ATLAS collaboration has observed photon-induced $W^{+} W^{-}$production in pp collisions using the full $13 \mathrm{TeV}$ dataset [8]. This production is interesting as at $\mathrm{LO}$ it only involves self-couplings of the $E W$ bosons. The main background consists of quark- and gluon-induced $W W$ production that is accompanied by hadronic activity, leading to a large number of associated tracks, contrary to signal events, where the two $\mathrm{W}$ bosons are isolated. Therefore, a precise description of the pile-up tracks and the track-multiplicity of the different processes is needed. The former is achieved with beam-spot size corrections for MC that mimic Run- 2 conditions and corrections for additional pp collisions obtained using $Z \rightarrow l l$ events. The description of the charge multiplicity of Drell-Yan and Diboson simulated events is improved using corrections derived from $Z \rightarrow l l$ events. It was observed that, despite being different processes, the track multiplicity of the three processes is similar, due to a similar final colour configuration. The signal was extracted from a profile-likelihood fit in the SR and multiple CRs, rejecting the background only hypothesis with 8.4 standard deviations. The distributions used for the calibration of the number of tracks and the final SR are shown in Figure 4.

Another analysis from the ATLAS collaboration reports the first observation of $W W W$ production [9], a process of great interest, as it is a direct probe of the strength of triple- and quartic-gauge couplings. The analysis exploits the leptonic decay of the $W$ boson, targeting the two same sign 

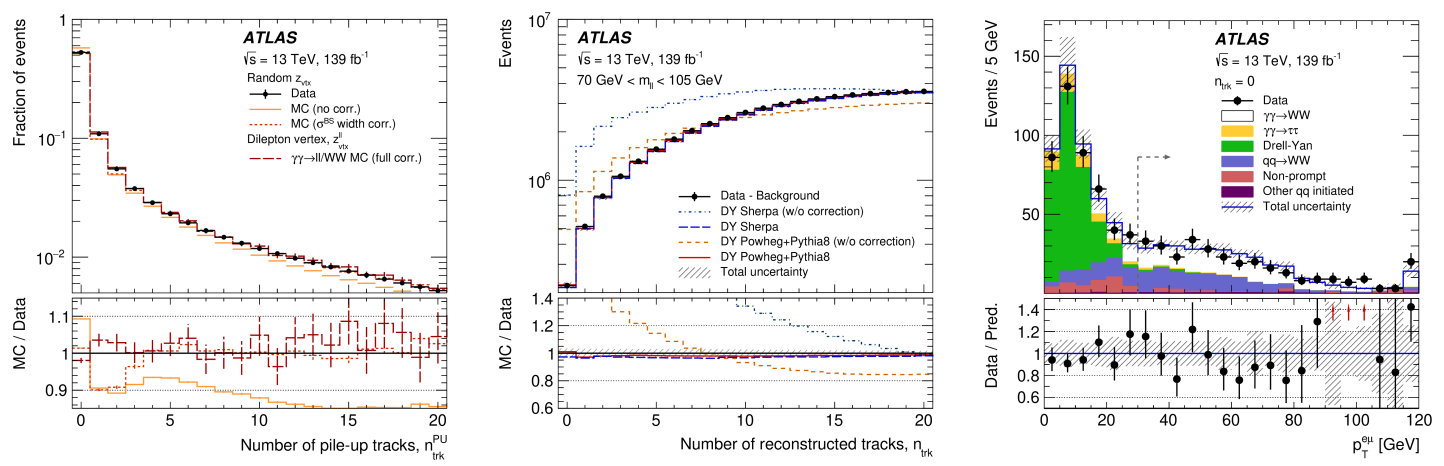

Figure 4: ATLAS: The density of pile-up tracks (left) and the number of reconstructed tracks in Drell-Yan events (central) compared to MC predictions at different stages of the calibration. The distribution of events in the signal region as a function of $p_{\mathrm{T}}^{e \mu}$ (right) [8].

lepton plus jets and the three lepton final states. The normalisation of the dominant $W Z+$ jets background process is obtained from three control regions defined by the number of jets as shown in Figure 5. Two BDTs are used to further improve the signal-to-background separation. As an example, the BDT output for the $\mu \mu$ and the three lepton SRs are shown in Figure 5. Finally, the signal strength is obtained from a binned maximum likelihood fit that uses the BDT distributions in the four SRs (ee, $e \mu, \mu \mu$ and three leptons) and the three CRs $m_{l l l}$ distributions. The $W W W$ production is observed with a significance of 8.2 standard deviations (where 5.4 standard deviations were expected).
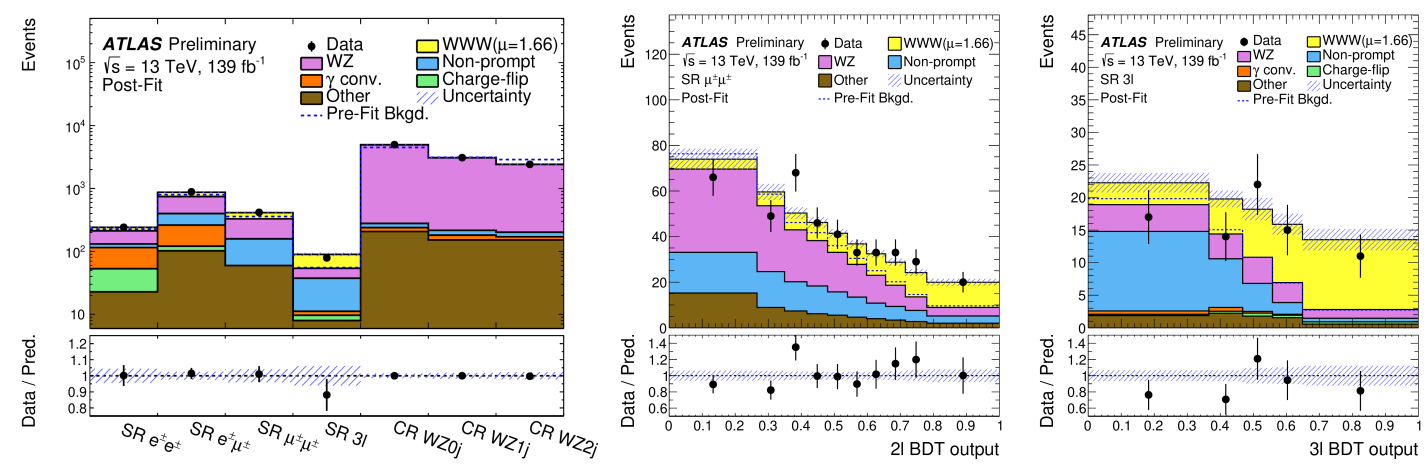

Figure 5: ATLAS: Event yields in the four signal regions and three control regions (left). The boosted decision tree output for the $\mu \mu$ (central) and the three lepton (right) channels[9].

\section{Properties measurements}

The measurement of particle properties provides also valuable information about the SM. In some cases, the measured properties are free parameters of the SM that have to be determined experimentally, for example the top quark mass. In other cases, the SM makes precise predictions, where measured deviations would directly point to new sources of physics, like a violation of the lepton flavour universality. 
The CMS collaboration has done a precise direct measurement of the invisible decay width of the $Z$ boson using a partial $13 \mathrm{TeV}$ dataset [10], targeting the $Z(v v)+$ jets production, competitive with the most precise direct measurement from the L3 Collaboration. Events used in the analysis are categorised into three main regions: the $Z(v v)+$ jets SR, and dilepton+jets $\left(Z, \gamma^{*}\right)$ and lepton+jets $(W)$ CRs, as shown in Figure 6. A correction as a function of the missing transverse momentum is derived for the dominant background process, $W+$ jets events in which the lepton is missing. The signal is extracted from a simultaneous fit to the SR and CRs. Additionally, the $Z, \gamma^{*} \rightarrow l l$ process is corrected to $Z \rightarrow l l$ using the MADGRAPH5_AMC@NLO program to extract the branching ratio. The measurement from the CMS collaboration in comparison to measurements from the LEP collaborations is shown in Figure 6.
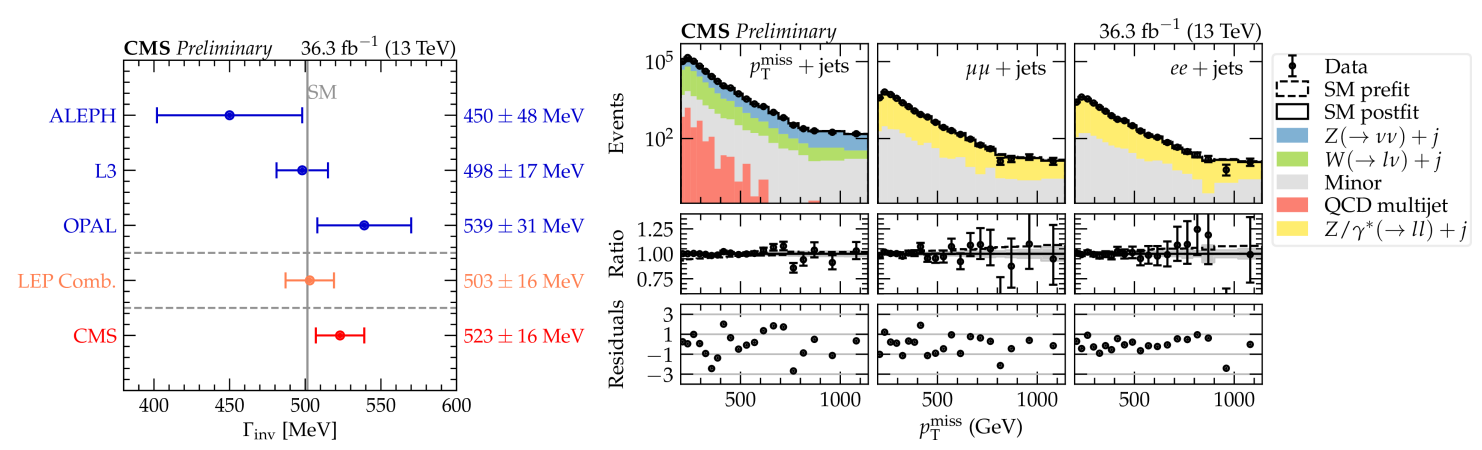

Figure 6: CMS: $\mathrm{Z}$ width into invisible particles compared to LEP measurements (left) and distributions of data and $\mathrm{MC}$ as functions of $p_{\mathrm{T}}^{\text {miss }}$ for signal and control regions (right) [10].

An important ingredient of the SM is that the couplings of the electroweak gauge bosons $(W, Z)$ to the charged leptons $(e, \mu, \tau)$ are independent of the lepton flavour. Using dileptonic $t \bar{t}$ events from the full $13 \mathrm{TeV}$ dataset, the ATLAS collaboration performed a test of the universality of $\tau$ and $\mu$ lepton couplings in $W$-boson decays [11]. This measurement is of particular interest because a previous measurement from the LEP collaborations shows some tension with the SM prediction. In this analysis, taus are identified from the muons of the decay $\tau \rightarrow \mu v_{\tau} \bar{v}_{\mu}$. As the two neutrinos carry a fraction of the original tau momentum, muons originating from a tau decay are, on average, less energetic than those from direct $W$-boson decays. Additionally, as the $\tau$-lepton has a significant lifetime compared to the $W$ boson, the subsequent muon appears at a displaced vertex. These two properties can be observed in Figure 7, where the predicted event yields of signal and background events are shown as functions of the transverse impact parameter $\left|d_{0}\right|$ of muons for two slices of the muon transverse momentum $p_{\mathrm{T}}^{\mu}$. The calibration of $\left|d_{0}\right|$ is improved using $Z \rightarrow \mu \mu$ events and the main background contributions are estimated using a CR around the $Z$ mass peak for the $Z \rightarrow \mu \mu+$ jets and same sign lepton CRs for muons from hadron decays. The result from the ATLAS collaboration exceeds the precision achieved by the LEP collaborations and is in agreement with the SM prediction, as shown in Figure 7.

\section{Effective field theory extension of the SM}

Finally, another way of testing the SM is to directly search for new physics in the LHC data by searching for deviations from the SM predictions. Such deviations can be interpreted in a coherent 

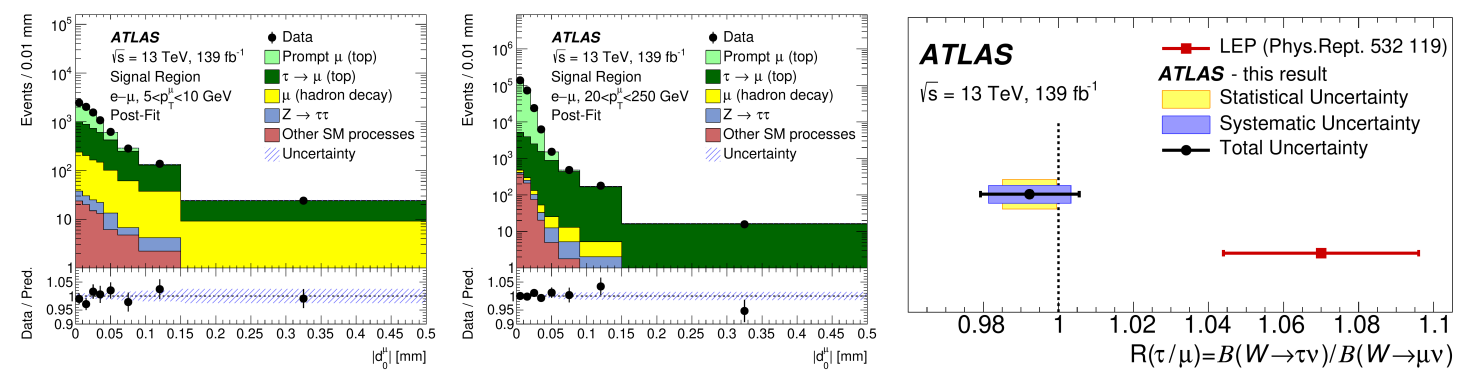

Figure 7: ATLAS: Distribution of $\left|d_{0}^{\mu}\right|$ for low $p_{\mathrm{T}}$ (left) and high $p_{\mathrm{T}}$ (central) muons and the measured ratio of the $W \rightarrow \tau v$ and the $W \rightarrow \mu v$ branching ratios in comparison to the SM prediction and the previous measurement from the LEP collaborations [11].

and model-independent manner by using the approach of effective field theory (EFT). The EFT corresponds to a low-energy limit of an underlying theory characterised by an energy scale $\Lambda$ far beyond the reach of current colliders.

Exploiting the rare production of top quarks with an associated Z boson, the CMS collaboration performed a search of new top quark interactions within the framework of an EFT using the full Run-2 dataset at $13 \mathrm{TeV}$ [12]. The analysis targets the production of $t \bar{t} Z, t Z q$ and $t W Z$ events to test an EFT extension of the $t \bar{t} Z$ vertex, where up to five dimension-six operators are considered. To make best use of the small signal sample, neural networks are extensively used in the analysis. First, a multiclass classifier divides the signal region into $t \bar{t} Z, t Z q$ and background subregions. Due to comparatively small event yields and the fact that it is kinematically close to $t \bar{t} Z$, the $t W Z$ signal is not targeted explicitly. Figure 8 shows the $t \bar{t} Z$ and $t Z q$ NN output nodes. Subsequently, binary classifiers are trained to separate SM events from scenarios, where at least one of the Wilson coefficients (WC) is non-zero. An example of such a classifier is shown in Figure 8. This technique is used to design observables with very high sensitivity to different WCs. The level of compatibility of the WCs with data is extracted from a simultaneous maximum likelihood fit to the data in six categories (four SR, two CR). The fit is done for individual WCs, for pairs of WCs and finally for all five WCs at the same time. All results are consistent with the SM at 95\% confidence level.
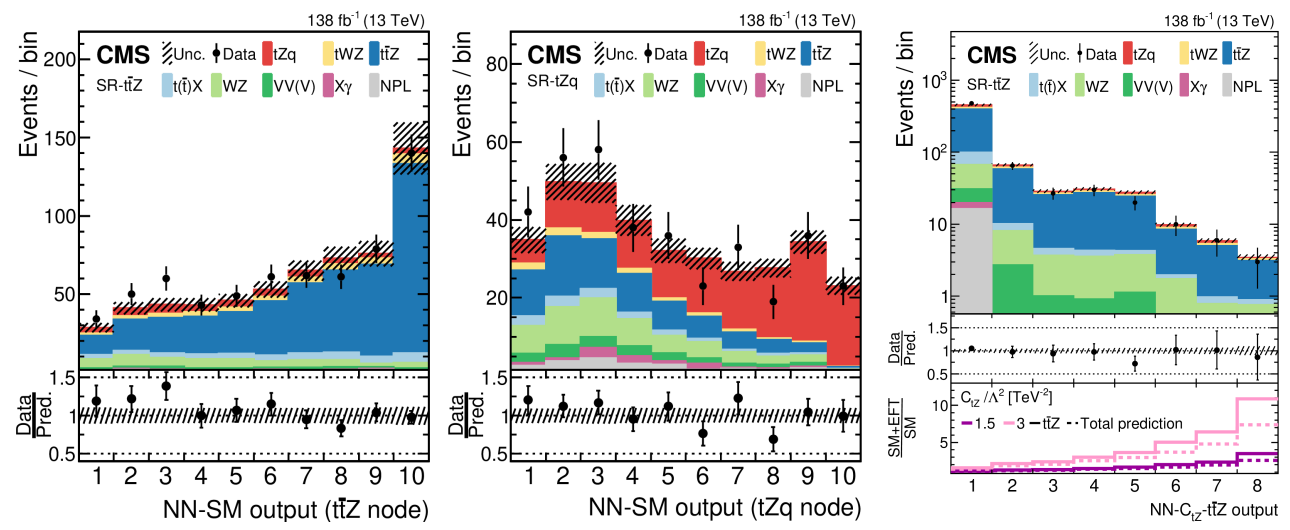

Figure 8: CMS: The $t \bar{t} Z$ (left) and $t Z q$ (central) multiclass neural network output nodes and the output of the binary neural network for the Wilson coefficient operator $C_{t Z}$ in the $t \bar{t} Z$ signal region (right) [12]. 


\section{Summary}

A selection of EW and QCD results from the ATLAS and CMS collaborations was presented, highlighting the variety of analysis strategies followed by both experiments. In the last years, as the complexity of the studied processes increased, the use of multivariate data analyses like neural networks became more relevant. These methods provide improvements in multiple applications, e.g. for signal-to-background discrimination or event reconstruction. Rare processes like the $W W W$ production were observed for the first time using the LHC Run-2 dataset. Despite the much more complex environment of proton-proton collisions at the LHC, some of the results are equally precise than results from the LEP collaborations. Despite all the effort, so far no significant deviations from the SM predictions have been found.

\section{References}

[1] L. Evans and P. Bryant (editors), LHC Machine, JINST 3 (2008) S08001.

[2] ATLAS Collaboration, The ATLAS experiment at the CERN Large Hadron Collider, JINST 3 (2008) S08003.

[3] CMS collaboration, The CMS experiment at the CERN LHC, JINST 3 (2008) S08004.

[4] J. Jiménez Peña, Highlights of EW and QCD results at the LHC, record of the talk.

[5] CMS collaboration, Measurement and QCD analysis of double-differential inclusive jet cross sections in pp collisions at $\sqrt{s}=13 \mathrm{TeV}$, CMS-PAS-SMP-20-011.

[6] ATLAS Collaboration, Measurement of the production cross section of pairs of isolated photons in pp collisions at $13 \mathrm{TeV}$ with the ATLAS detector, CERN-EP-2021-105, arXiv:2107.09330 [hep-ex].

[7] CMS collaboration, Inclusive and differential cross section measurements of single top quark production in association with a $\mathrm{Z}$ boson in proton-proton collisions at $\sqrt{s}=13 \mathrm{TeV}$, CMSPAS-TOP-20-010.

[8] ATLAS Collaboration, Observation of photon-induced $W^{+} W^{-}$production in pp collisions at $\sqrt{s}=13$ TeV using the ATLAS detector, Phys. Lett. B 816 (2021) 136190.

[9] ATLAS Collaboration, Observation of $W W W$ production in pp collisions at $\sqrt{s}=13 \mathrm{TeV}$ with the ATLAS detector, ATLAS-CONF-2021-039.

[10] CMS collaboration, Precision measurement of the $Z$ invisible width with the CMS experiment in pp collisions at $\sqrt{s}=13 \mathrm{TeV}$, CMS-PAS-SMP-18-014.

[11] ATLAS Collaboration, Test of the universality of $\tau$ and $\mu$ lepton couplings in W-boson decays with the ATLAS detector, Nat. Phys. 17 813-818 (2021).

[12] CMS collaboration, Probing effective field theory operators in the associated production of top quarks with a $Z$ boson in multilepton final states at $\sqrt{s}=13 \mathrm{TeV}$, CMS-TOP-21-001, arXiv:2107.13896 [hep-ex]. 